\title{
First-Principles Simulations of an Aqueous CO/Pt(111) Interface
}

Lan, Jinggang ; Hutter, Jürg ; Iannuzzi, Marcella

DOI: https://doi.org/10.1021/acs.jpcc.8b05933

Posted at the Zurich Open Repository and Archive, University of Zurich ZORA URL: https://doi.org/10.5167/uzh-168889

Journal Article

Accepted Version

Originally published at:

Lan, Jinggang; Hutter, Jürg; Iannuzzi, Marcella (2018). First-Principles Simulations of an Aqueous CO/Pt(111) Interface. Journal of Physical Chemistry C, 122(42):24068-24076.

DOI: https://doi.org/10.1021/acs.jpcc.8b05933 


\title{
First-Principles Simulations of Aqueous $\mathrm{CO} / \mathrm{Pt}(111)$ Interface
}

\author{
Jinggang Lan, Jürg Hutter, and Marcella lannuzzi* \\ Department of Chemistry, University of Zürich, Zürich \\ E-mail: marcella.iannuzzi@chem.uzh.ch \\ Phone: +41446354479
}

\begin{abstract}
We discuss the structural, electronic and vibrational properties of an adsorbed CO monolayer in the presence of liquid water at room temperature, as obtained by abinitio molecular dynamics simulations. The water molecules at closest distance from the substrate form a bi-layer, where they are co-adsorbed at empty surface sites and with relatively rigid orientation, at the difference with the bulk liquid above. Co-adsorbed water strengthens the back-bonding to $\mathrm{CO}$, which leads to the red shift of about 40 $\mathrm{cm}^{-1}$. The synergy between the first bi-layer and the bulk liquid induce the further polarization of the $\mathrm{CO}$ bond, which changes the transition dipole and results in the $\mathrm{CO}$ intensity enhancement. It is also verified that no hydrogen bond is effectively present between $\mathrm{CO}$ and bulk water. These findings provide new insight into the physics of the surface/adsorbate/solvent interface, thus clarifying the experimental observation, to be exploited for the design of improved catalysts.
\end{abstract}




\section{Introduction}

Most of the relevant processes in an electrochemical cell occur at solid/liquid interfaces. Unfortunately, the direct observation of these processes and the characterization of the interfaces under operando conditions are still challenging for both experimental and modelling approaches. ${ }^{1-4}$ The fundamental understanding of interfaces at the atomic level would greatly help in predicting the stability and reactivity of materials and eventually would lead to the rational tuning of the different components of heterogeneous catalysts. This is the reason why significant efforts are directed in investigating adsorption and dynamics of adsorbates at metal and semiconductor surfaces in contact with electrolytes. ${ }^{1-8}$

Several vibrational spectroscopic techniques, such as surface-enhanced infra-red spectroscopy, ${ }^{9}$ sum-frequency generation spectroscopy, ${ }^{10,11}$ two dimensional spectroscopic techniques $^{11,12}$ and attenuated total reflection infra-red spectroscopy (ATR-IR) ${ }^{12-14}$ can be applied in detecting changes in molecular structure and environment. However, both surface sensitivity, to acquire data specifically from the very small portion of interest in a macroscopically large condensed matter system, and time resolution, to follow and characterize reaction processes, are in general not easy to achieve. ATR-IR spectroscopy is ideally suited for studying molecular vibrations at the solid-liquid interface because the infra-red light is restricted close to the catalyst surface minimizing the contribution of the bulk liquid phase to the IR spectrum. ${ }^{15}$ Platinum is very often used as electrode or catalyst in electrochemical cells, thanks to its catalytic activities and excellent reproducibility. The adsorption of carbon monoxide $(\mathrm{CO})$ on platinum surfaces in aqueous conditions is of great interest because of its industrial application in the field of electro-chemistry. Moreover, thanks to the very specific vibrational properties, CO molecules are often employed as a molecular label. The apparently simple and well-established system, however, involves an underlying physical complexity associated with the dynamic nature of solvent and adsorbates at the surface.

The adsorption of $\mathrm{CO}$ on $\mathrm{Pt}(111)$ is regarded as a relatively simple model system that can be employed to study fundamental aspects related to variations of the characteristic 
the spectroscopic signatures due to the environment effects. ${ }^{16-20} \mathrm{CO}$ is known to chemisorb linearly on surface Pt atoms. ${ }^{21}$ Bridge adsorption geometries are slightly less stable, and can be identified by significantly shifted bands in the IR spectrum. ${ }^{13,14,19,20}$ On the other hand, it has been experimentally observed that the presence of liquid water modifies measurably the adsorption of $\mathrm{CO}$ on platinum, inducing a red shift and the intensity enhancement of the stretching mode of the linearly bound $\mathrm{CO}$, together with a decreased ratio between linear and bridge adsorption geometries. The increased back-donation from metal and a direct interaction with water molecules, i.e., hydrogen bonding, have been proposed as possible causes of the observed spectral differences with respect to the dry conditions. ${ }^{13,14}$ Two-dimensional spectroscopic techniques are able to measure different types of spectral correlations, and using a pump and probe approach can provide information on the evolution over the time scales determined by the separation between the pulses. ${ }^{22}$ Kraack et al. ${ }^{12}$ show a predominant asymmetric band at $2050 \mathrm{~cm}^{-1}$ and a weak absorbance signal at $1890 \mathrm{~cm}^{-1}$ and also conclude that the $\mathrm{CO}$ molecule mostly binds linearly to $\mathrm{Pt}$ also in aqueous solution, while no measurable CO-CO coupling is revealed. By resolving the 2D line shape of Pt-CO, Xiong et al. ${ }^{11}$ find that there is a significant inhomogeneous contribution, suggesting the presence of a slow component to the vibrational dynamics. Heterogeneity could be caused by some combination of surface crystallinity and weak hydrogen bonding of water to CO.

In the present work, we address the effect of coverage and of liquid water environment on the adsorption of $\mathrm{CO}$ at the $\mathrm{Pt}(111)$ surface by means of ab-initio molecular dynamics (AIMD) simulations. By monitoring the structural and dynamic properties of water close to the interface and their correlation to the spectral changes, we are able to answer some open questions regarding this solid/liquid interface, ${ }^{9-11,13-15,19-21} \mathrm{e.g}$, the origin of the red-shift and intensity enhancement of the CO stretching mode, ${ }^{9,13-15}$ as well as phenomena associated to the coupling among adsorbates. ${ }^{10,12,2311,13,14}$

It is important to notice that the specific structural properties addressed here are determined by the complex interactions between the metallic surface and the co-adsorbed water 
and CO molecules. Static models ${ }^{24-27}$ are unsuitable because they do not capture many-body effects, dynamics of the adsorbates, anharmonicities, temperature effects and an explicit polarizable environment. Moreover the resulting spectral signatures are a consequence of the electronic redistribution and of the related reactivity of the metal surface, which are hard to capture with more simplistic classical force field approaches. Indeed, both the formation of the water bi-layer as well as the liquid water rearrangement upon CO co-adsorption could not be captured by MD simulation driven by classical force fields. ${ }^{28}$ The choice of AIMD is then mandatory for a sufficiently accurate description of the interaction between water molecules and carbon monoxide adsorbed on the platinum surface. Indeed, AIMD has been applied already to study analogous water/metal interfaces. ${ }^{29-32}$ In our work we show that the scope of AIMD can be significantly extended by employing the second generation Car-Parrinello molecular dynamics (SGCP) scheme. ${ }^{33-35}$ Thus we propose a simulation protocol that allows the efficient sampling over long time scales, also for metallic systems and when the explicit solvent is included. This is obviously a significant advancement since we demonstrate that with the proposed approach the understanding of the interactions is strongly improved and can be exploited for the interpretation of the experimental findings.

\section{Methods}

All simulations are carried out by means of the $\mathrm{CP} 2 \mathrm{~K}^{36}$ program package. The electronic structure is described at the Kohn-Sham density functional level of theory, within the Gaussian and plane waves (GPW) ${ }^{37}$ framework. The molecular orbitals of the valence electrons are expanded into DZVP-MOLOPT-GTH basis sets, ${ }^{38}$ while the interaction with the cores is described through Goedecker-Teter-Hutter (GTH) pseudopotentials. ${ }^{39,40}$ The efficient solution of the Poisson equation within the $\mathrm{PBC}$ is obtained in the reciprocal space by the expansion of the electronic density into a plane-wave basis set truncated at the energy cut-off of 500 Ry. The resulting orthorhombic simulation cell has dimensions $14.93 \times 17.24 \times 57$ 
$\AA^{3}$. All simulations are carried out by keeping the two bottom Pt layers constrained at the initial coordinates in order to maintain the bulk behaviour of the inner part of the slab. This strategy is justified by the fast decay of the $\mathrm{Pt} /$ adsorbate interaction.

For the choice of the exchange and correlation functional, we have considered the accuracy in the description of the adsorbed state of the $\mathrm{CO}$ molecule on $\mathrm{Pt}(111)$ and of the bulk phase of liquid water. As mentioned above, previous studies have demonstrated that $\mathrm{CO}$ is linearly bounded on Pt, at least at low coverage. However, structure optimisations at the DFT level of theory give contradictory results depending on the choice of the functional, since some of them predict bridged/multi-bound configurations as most stable. ${ }^{16,41-47}$ (Details in SI). We choose the BLYP functional ${ }^{48,49}$ which is able to reproduce the right hierarchy among the $\mathrm{CO}$ adsorption configurations, and augmented with the Grimme's D3 dispersion corrections ${ }^{50}$ equilibrates liquid water at the density of $1.07 \mathrm{~kg} / \mathrm{m}^{3}$, i.e., in acceptable agreement with the experimental conditions and previous calculations. ${ }^{51,52}$

DFT-based MD simulations are most commonly carried out according to the BornOppenheimer procedure, i.e., by optimizing the electronic structure at each step along the integration of the equations of motion for the atomic coordinates. This protocol can be significantly accelerated by properly propagating the electronic density by ad hoc extrapolation schemes. However, when metallic electronic structures are involved, the efficiency of the electronic structure optimization as well as of the extrapolation algorithms rapidly degrades, due to the infamous charge sloshing problem. The convergence difficulties become even worse by increasing the system size, due to the divergent eigenvalue spectrum of the charge dielectric function of metallic systems. This is the reason why AIMD simulations of metals are often excessively demanding. In order to achieve an extensive sampling by MD, our strategy is to apply the second generation Car-Parrinello molecular dynamics method, ${ }^{33-35}$ which has proven to be accurate and efficient for the extended sampling of large condensed matter systems, in particular when metallic electronic structures are involved. The SGCP method has been developed to get the best out of both CP-MD and BO-MD. As BO-MD, it allows 
for large integration time-steps, it includes an adaptive control of deviations from the BO surface, and it can handle systems with vanishing band-gap. On the other hand, it does not rely on an iterative self-consistent wave-function optimization scheme, as CP-MD. In spite of this, the SGCP is also accurate, as its dynamics is almost indistinguishable from BOMD, providing that the proper settings are used. ${ }^{33,34}$ For the propagation of the electronic degrees of freedom, the always stable predictor corrector scheme by Kolafa ${ }^{53}$ is set at the order $k=2$. The correction step is obtained by 5 iterations of the orbital transformation optimisation algorithm. ${ }^{54}$ Our simulations run SGCP in the canonical ensemble at 300K, propagating the equations of motion according to the Langevin dynamics, with a time step $\Delta \mathrm{t}=0.5 \mathrm{fs}:$

$$
\begin{aligned}
M_{I} \ddot{\mathbf{R}}_{I} & =\mathbf{F}_{\mathrm{BO}}-\left(\gamma_{D}+\gamma_{L}\right) \dot{\mathbf{R}}_{I}+\boldsymbol{\Xi}_{I} \\
& =\mathbf{F}_{\mathrm{ASPC}}-\gamma_{L} \dot{\mathbf{R}}_{I}+\boldsymbol{\Xi}_{I}
\end{aligned}
$$

where $\boldsymbol{\Xi}_{I}=\boldsymbol{\Xi}_{D}+\boldsymbol{\Xi}_{L}$ is a random noise obeying the fluctuation and dissipation theorem, $M_{I}$ is the ionic mass, $\gamma_{L}$ is the Langevin friction coefficient, and $\gamma_{D}$ is the intrinsic friction coefficient to control the dissipation in SGCP. $\gamma_{L}$ is set at $0.001 \mathrm{fs}^{-1} \cdot \gamma_{D}$ has been adjusted by running pre-equilibration simulations where the temperature and the conservation of the total energy have been monitored. It turns out that for a proper thermalisation of the system at $300 \mathrm{~K}$, the $\gamma_{D}$ parameter has to be kind-dependent, i.e., we use $5 \times 10^{-5} f s^{-1}$ for Pt, $5 \times 10^{-5} f s^{-1}$ for $\mathrm{CO}, 2.2 \times 10^{-4} f s^{-1}$ for $\mathrm{H}_{2} \mathrm{O}$.

The IR spectra are obtained from the Fourier transform of the dipole autocorrelation function

$$
A(\omega) \propto \int\langle\mu(\tau) \mu(t+\tau)\rangle_{\tau} e^{-i \omega t} d t
$$

using the program package TRAVIS. ${ }^{55,56}$ Assuming that the MD sampling is properly extended, this approach accounts for the contributions from all statistically relevant configu- 
rations, including anharmonic effects.

Within periodic boundary conditions, the total electric dipole moment $\mu$ is evaluated using the Berry phase scheme. ${ }^{57-59}$ With a side length $L$ and considering only the $\Gamma$ point in the Brillouin zone, each component of the electric-dipole moment is then

$$
\mu_{\alpha}=\frac{e}{2 \pi} \operatorname{Im} \ln \operatorname{det} \mathbf{S}^{(\alpha)}
$$

where the matrix $\mathbf{S}$ is defined using the Kohn-Sam orbitals $\phi_{n}$

$$
S_{n, m}^{(\alpha=x)}=\int_{L} \phi_{n}^{*}(x) \exp \left[-i \frac{2 \pi}{L} x\right] \phi_{m}(x) d x
$$

The Berry phase scheme is used also for the nuclear dipole, by assigning the effective core charges to the instantaneous nuclear positions.

\section{Results and discussions}

The ball and stick representation of the $\mathrm{CO} / \mathrm{Pt}(111)$ system in the vacuum and covered by a liquid water film is shown in Fig. 1. The metal slab consists of four $(6 \times 6)(111)$ atomic layers. Four different $C O$ coverages have been simulated, i.e., $\Theta=0,0.11,0.25$, 0.50. The $\mathrm{CO}$ molecules are initially adsorbed in the linear configurations since previous experimental and theoretical studies have shown that bridged configurations are less stable on $\mathrm{Pt}(111) .{ }^{21}$ In order to reproduce the proper solvation environment, a film of 134 water molecules, corresponding to a thickness of approximately $18 \AA$, is located on the surface. Since periodic boundary conditions are applied in all three dimensions, a vacuum region of

$30 \AA$ is introduced in order to decouple the periodic images in the direction perpendicular to the slab.

Our discussion on the interface's properties is based on the analysis of SGCP production trajectories of $30 \mathrm{ps}$, which are sufficiently long to guarantee the convergence of the relevant 


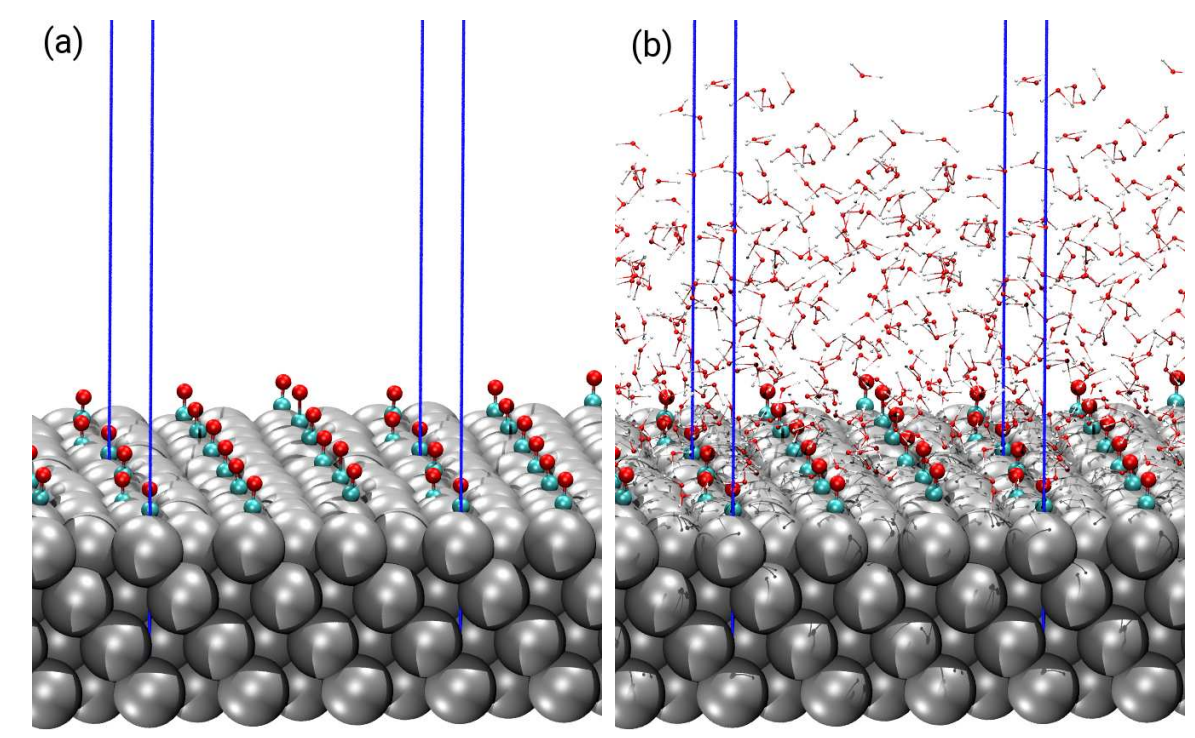

Figure 1: Atomistic model representing (a) the monolayer of $\mathrm{CO}$ adsorbed on the $\mathrm{Pt}(111)$ slab in vacuum and (b) the same $\mathrm{CO} / \mathrm{Pt}$ slab in contact with liquid water. The blue lines delimit the simulation cell in periodic boundary conditions. Color code: silver spheres are $\mathrm{Pt}$ atoms, cyan $\mathrm{C}$ atoms, red $\mathrm{O}$ atoms, and grey $\mathrm{H}$ atoms.

correlation functions, as the dipole autocorrelation function for the calculation of the IR spectrum.. We compare four different $\mathrm{CO}$ coverages. The clean $\operatorname{Pt}(111)$ surface $(\Theta=0)$ is the reference system for the characterization of the water-metal interaction. The rearrangement of liquid water at this interface is well illustrated by the modifications in the averaged atomic density distributions of $\mathrm{H}$ and $\mathrm{O}$ atoms. The plot in Fig. 2 shows the profile of the density along the normal to the surface ( $z$ Cartesian axis), computed as the average density of water located at a given height from the surface, within an interval of $0.1 \AA$. The average position of the surface Pt atoms is set at zero. The density profile shows a first structured layer at $1.30 \AA$, closely followed by the second maximum at 2.2 and then a third at $4.3 \AA$. For a better characterization of this interface, we have investigated in more details the water structure as a function of the distance from the metal. In particular, we have computed the distribution of the $\mathrm{O}-\mathrm{H}$ bond length and the orientation of the water molecule with respect to the surface normal, as displayed in the Fig. 3. The orientation is defined in terms of the angle between the $\mathrm{OH}$ bond and the normal to the surface, i.e., cosines close to +1 
correspond to $\mathrm{OH}$ pointing outwards (to bulk), cosines around 0 correspond to $\mathrm{OH}$ parallel to the surface, negative values indicate $\mathrm{OH}$ pointing towards the surface. As it is expected, in the bulk region, between 6 and $14 \AA$ from Pt, the $\mathrm{O}-\mathrm{H}$ bond fluctuates around $0.99 \AA$, with a variance of $0.10 \AA$, and no orientation is preferred, being the cosines homogeneously distributed between -1 and +1 . At the two opposite interfaces, with Pt, between 1 and $6 \AA$, and with vacuum, between 14 and $20 \AA$, the behaviour of the liquid changes.

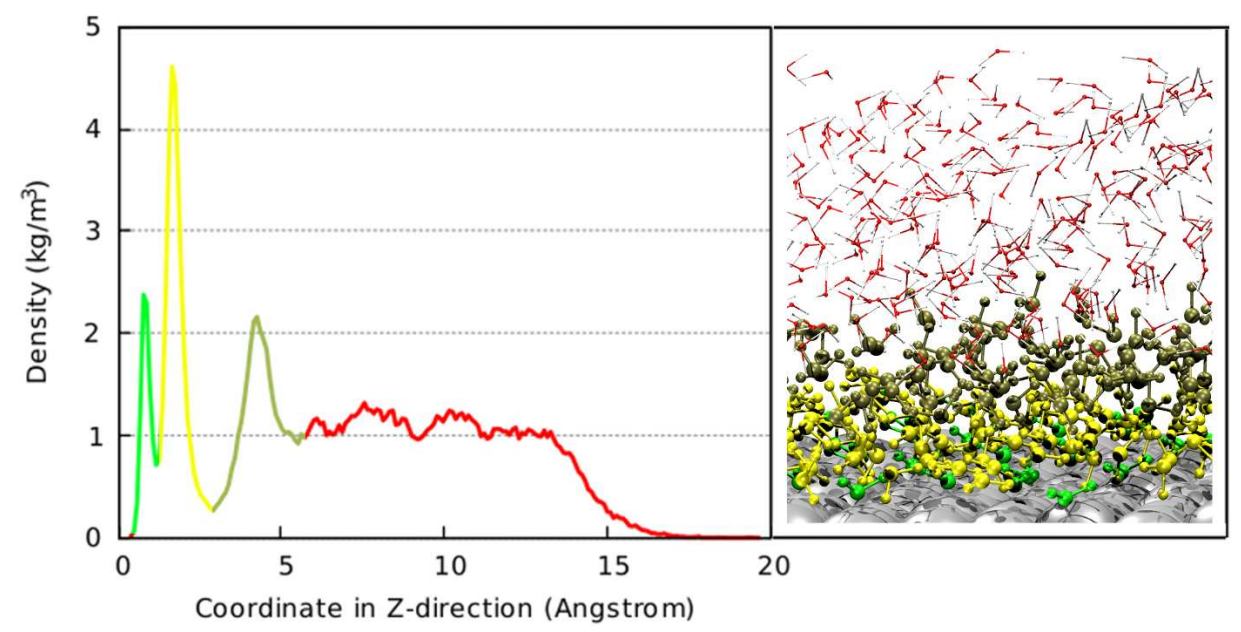

Figure 2: Left: Density profile of water along the normal to the surface. Right: snapshot extracted from the AIMD trajectory. Water molecules belonging to different layers, i.e., at different height from Pt(111), are distinguished by colour: green denotes molecules in the first layer, yellow in the second layer, tan in the third layer, while red/white lines are used for all other molecules. The same colour code is used in the atomic density plot, to highlight the contributions from the different layers.

Close to the $\mathrm{Pt}(111)$ surface, the mobility of the water molecules is hindered due to the interaction with the metal. The self-diffusion coefficient obtained from the trajectory (See Fig.S4 in Supporting Information) based on Einstein relation is about $2.36 \times 10^{-9} \mathrm{~m}^{2} / \mathrm{s}$ in the bulk, in agreement with the experimental value ${ }^{60}$ of $2.57 \times 10^{-9} \mathrm{~m}^{2} / \mathrm{s}$, while for the bi-layer close to the surface it is reduced to $0.38 \times 10^{-9} \mathrm{~m}^{2} / \mathrm{s}$. Indeed, within the first water layer, the molecules are chemisorbed at the surface. The most probable adsorption configuration is with the $\mathrm{O}$ atom atop a $\mathrm{Pt}$ atom, slightly elongated $\mathrm{OH}$ bonds, which lay with outwards angles between $60^{\circ}$ and $80^{\circ}$, in agreement with previous studies. ${ }^{61}$ The water molecules of 

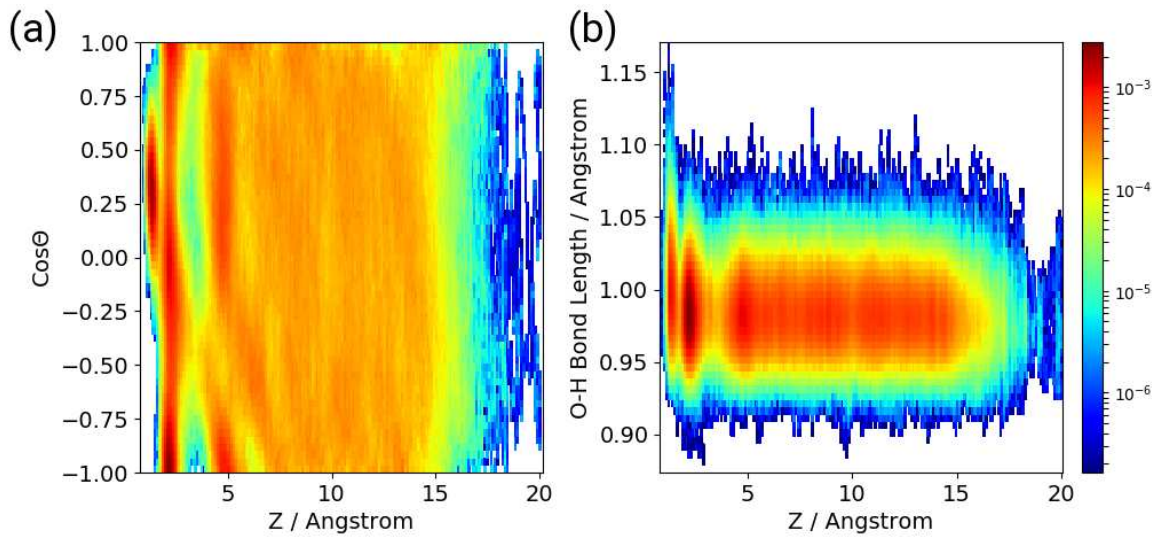

Figure 3: Distribution of (a) the intra-molecular $\mathrm{O}-\mathrm{H}$ bond length and (b) of the cosine of the angle between $\mathrm{OH}$ and the surface normal, plotted as functions of the height over the clean $\mathrm{Pt}(111)$ surface. The colour code from blue to red indicates increasing probability of finding the respective value. The distributions are obtained as averages over all $\mathrm{OH}$ and the whole trajectory.

the second layer show only a slight preference for downwards $\mathrm{OH}$ orientation, i.e. almost perpendicular to the surface (negative $\cos \theta$ ), which is associated to the interaction of the H-end with a hollow site. The resulting bi-layer presents a quite ordered pattern, as shown in Fig. S5 in Supporting Information, i.e., an ice-like character. ${ }^{29,30}$ The liquid-like behaviour is recovered farther from the surface, beyond a depletion region of more than $1.5 \AA$. Here the density distribution is broader and shallower, corresponding to about $1.07 \mathrm{~kg} / \mathrm{m}^{3}$, and by the pair distribution function, $g_{o o}(r)$, shows the characteristic features of liquid water (see Fig. S2 in Supporting Information). Exchanges of molecules between the structured bi-layer at the surface and the bulk liquid are rare events that have not been significantly sampled along our trajectories (see Fig. S3 in Supporting Information). Beyond $15 \AA$, the density decays to zero at the water/vacuum interface (structural details in Fig. 3, Fig. S1 in Supporting Information and ref. $\left.{ }^{62}\right)$.

The structural features of the CO adsorption, both in vacuum and with the liquid environment, are well illustrated by the bond length distributions plotted in Fig. 4. At low CO coverage of $\Theta=0.11$, only one type of adsorption mode is observed, where each molecule forms a linear bond via the $\mathrm{C}$-end on top of a surface $\mathrm{Pt}$ atom. The correlated $\mathrm{C}-\mathrm{O}$ and 
C-Pt distance distributions shown in panels (a) and (d) of Fig. 4 confirm the stability of the atop configuration in vacuum and in liquid environment, respectively. In vacuum, the average $\mathrm{Pt}-\mathrm{C}$ distance is $1.864 \AA$ and the average $\mathrm{C}-\mathrm{O}$ bond is $1.144 \AA$ long. At the aqueous interface, the average $\mathrm{C}-\mathrm{O}$ bond is slightly elongated $(1.151 \AA)$ and the $\mathrm{Pt}-\mathrm{C}$ distance is slightly shorter (1.849 $\AA$ ). From the water density plot in Fig. 5, we also observe that the characteristic water bi-layer at the interface is still present, even though it contains a reduced number of molecules because some adsorption sites are occupied by $\mathrm{CO}$. The $\mathrm{O}$ atoms of the first water layer are closer to the surface than the $\mathrm{C}$ atoms, while the $\mathrm{OH}$ bonds are still oriented between parallel and slightly outwards. The $\mathrm{O}$ atoms of the second water layer are lower than the O-end of the $\mathrm{CO}$ adsorbates, which is then within the depletion layer of the water film.
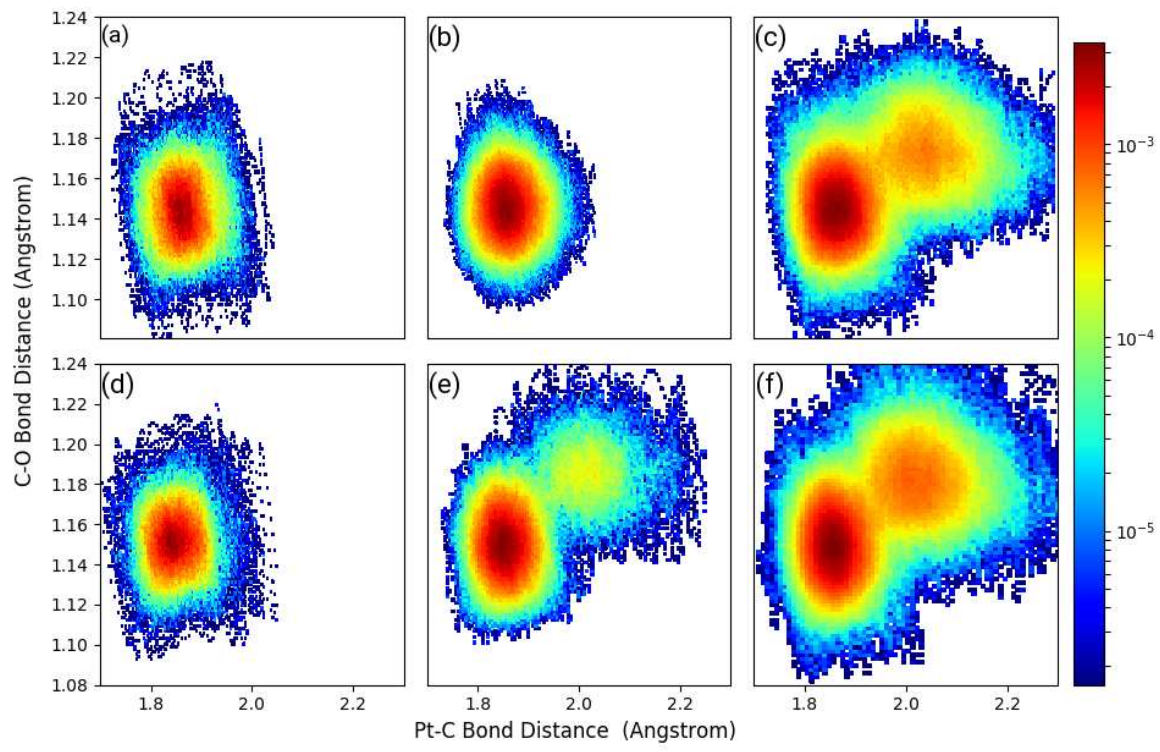

Figure 4: $\mathrm{C}-\mathrm{O}$ vs $\mathrm{Pt}-\mathrm{C}$ bond length probability distribution obtained from the MD sampling at different coverages in vacuum (top panels) and in liquid water (bottom panels). a) and d) are for $\Theta=0.11, \mathrm{~b}$ ) and e) for $\Theta=0.25$, and c) and f) for $\Theta=0.50$. The probability is represented with a logarithmic scale.

At the higher coverage of $\Theta=0.25$, the atop geometry is still the only one observed in vacuum, where neither hopping events nor intermediate adsorption configurations at bridge 
or hollow sites are observed. The Pt-C bond length fluctuates between 1.75 and $2.00 \AA$, while the $\mathrm{C}-\mathrm{O}$ bond is between 1.10 to $1.20 \AA$. On the other hand, in liquid water environment, besides atop adsorption, both bridge and hollow configurations can be observed. While the hollow site is only short-lived, being a transient configuration occurring along a hopping event between two bridge sites, the bridge adsorption site can be rather stable. This is in agreement with the experimental observation of a downward shift of the linear-to-bridge ratio from 6.9 to $4.1 .^{14}$ The molecules occupying bridge sites are characterized by a longer $\mathrm{C}-\mathrm{O}$ bond, as well as a longer average $\mathrm{Pt}-\mathrm{C}$ distance. Hence, their presence appears in the bond-length distribution plot as a second smaller peak, while the atop distribution gets narrower (see middle panel of Fig. 4). In the bridge configuration the two Pt-C distances are longer, but the distance of the molecule from the surface plane is reduced, as shown from the formation of double peaks in the density distributions. Another evident consequence of the $\mathrm{CO}$ coverage increase is that the structured water bi-layer is progressively modified, inducing the attenuation of the first density peak as the number of the chemisorbed water molecules is further reduced.

While for $\Theta=0.25$ only a small portion of $\mathrm{CO}$ molecules are found in bridged geometry and the hopping events are solely induced by the presence of the co-adsorbed water, the probability increases significantly at higher coverage, also in vacuum. At a coverage of half a monolayer $(\Theta=0.5)$, the two configurations become almost equally probable and the bond length fluctuations are significantly larger. At this coverage the adsorbates interact repulsively and become less stable, leading to a disordered pattern. The characteristic water bi-layer structure has almost vanished, since the numerous $\mathrm{CO}$ adsorbates prevent water from approaching closely the Pt surface, thus suggesting that the enhanced CO mobility is due to the direct or indirect (through Pt) CO-coupling effects.

From an experimental point of view, this same interface has been investigated by means of infra-red spectroscopy. Comparing the spectra of $\mathrm{CO} / \mathrm{Pt}(111)$ in the vacuum and in liquid water, a red shift of about $35 \mathrm{~cm}^{-1}$ and a fourfold enhancement of the $\mathrm{CO}$ stretching 


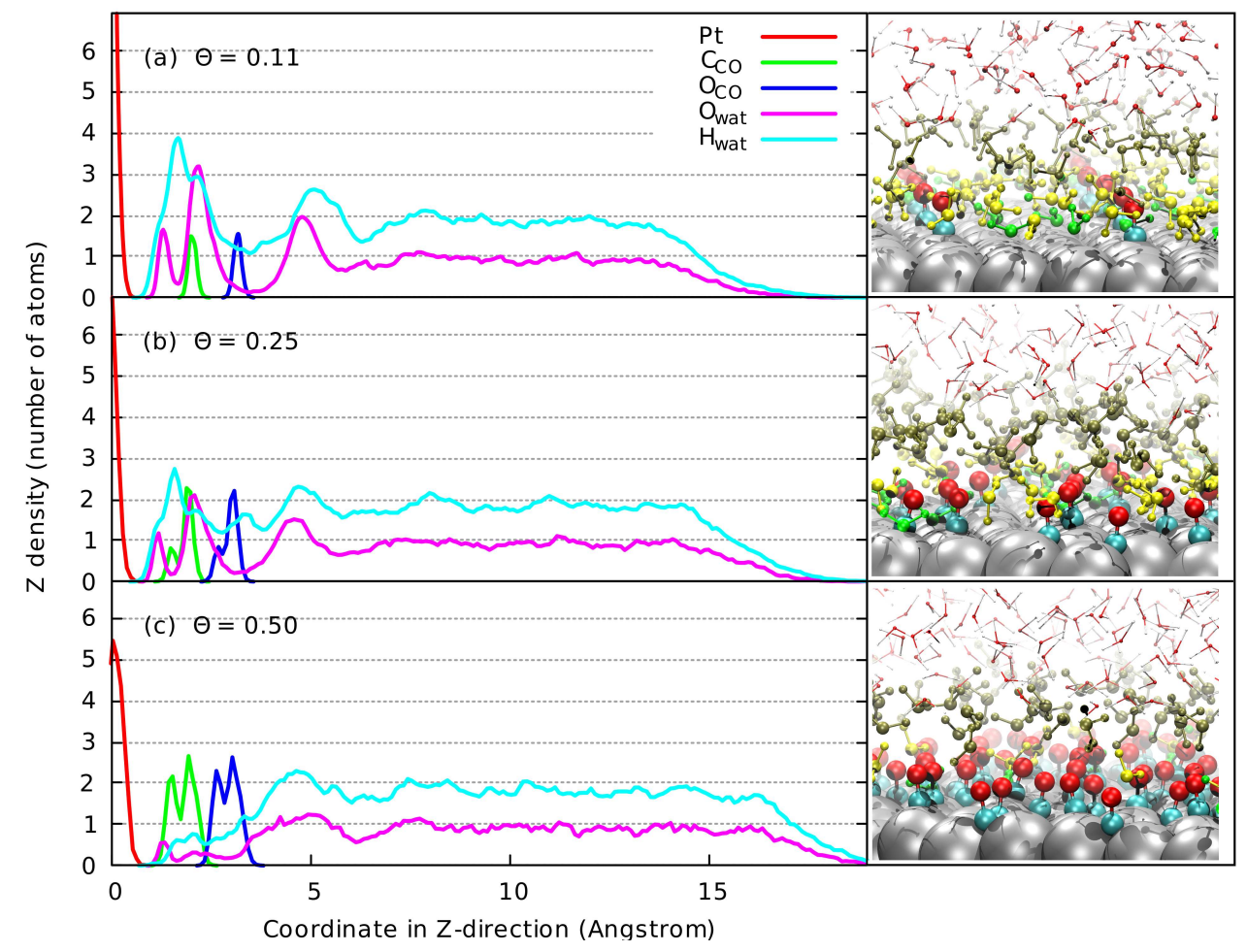

Figure 5: Left: Atomic density profile computed for each individual species along the normal to the surface, at the four different coverages, (a) $\Theta=0.11$, (b) $\Theta=0.25$, and (c) $\Theta=0.50$. Right: representative snapshots extracted from the trajectories generated with the three different CO coverages. Colour code as in Fig. 2. 
signal have been observed. This effect has been attributed to some form of coupling between the linearly bound adsorbates and the liquid environment, while the possible presence of multi-bond geometries seem not to play a relevant role in this respect. ${ }^{12}$

In order to verify how the different coverages and the corresponding liquid water restructuring at the interface might affect the vibrational properties of the adsorbates, we calculate the IR spectra from the generated trajectories. The spectra are obtained from the Fourier transform of the dipole autocorrelation function as described in Eq.3.

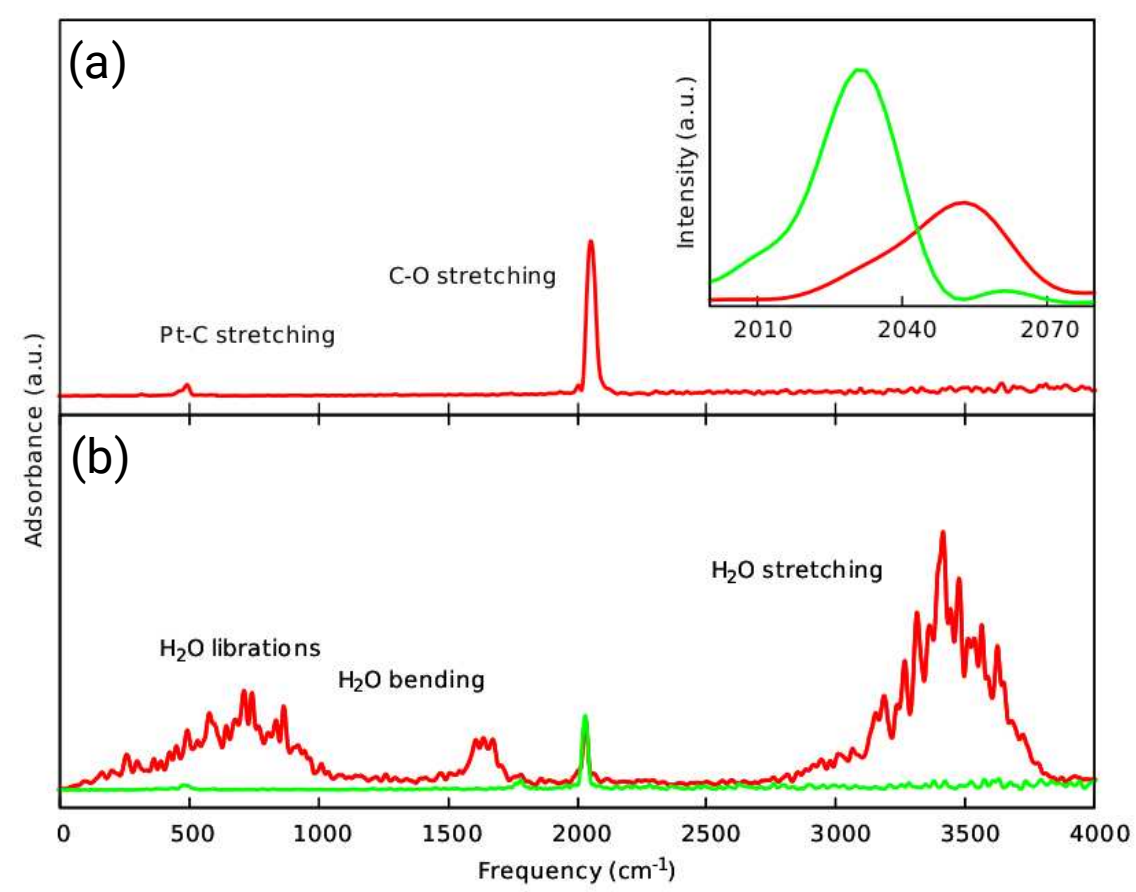

Figure 6: $\mathrm{CO} / \mathrm{Pt} \mathrm{IR}$ spectra calculated at the coverage of $\Theta=0.25$, in vacuum (a) and water (b). In (b) the green line represents the spectrum obtained considering only the $\mathrm{CO} / \mathrm{Pt}$ subsystem, i.e., by subtracting the liquid water contribution. Top-right: zoom-in of the region around the $\mathrm{CO}$ stretching mode, where the spectra without (red) and with (green) liquid water are directly compared.

As discussed above, with coverage $\Theta=0.25$ in vacuum the $\mathrm{CO}$ molecules remain stably bound at atop sites. The resulting IR spectrum plotted in Fig. 6 (top panel) shows a narrow $\mathrm{CO}$ stretching band at $2052 \mathrm{~cm}^{-1}$ and a much less intense signal from the PtC stretching at $495 \mathrm{~cm}^{-1}$. No significant effects of vibrational coupling due to the adsorbate-adsorbate 
interaction are revealed from our calculations, in agreement with experiment. ${ }^{12}$

When the system is solvated by water, a few hopping events are observed, corresponding to atop-to-bridge diffusion or bridge-to-bridge diffusion. These processes are activated by the interaction of the metal with water. In particular, some molecules approach the surface and occupy some of the available adsorption sites, thus perturbing the CO layer. Nevertheless, the large majority of the CO molecules are found at atop sites. Therefore, the IR spectrum (Fig. 6 bottom panel) is strongly dominated by their contribution. Moreover, the signal due to the bridged $\mathrm{CO}$ molecules overlaps with the water bending band, hence cannot be easily distinguished. More interestingly, the presence of the liquid induces a $\sim 20 \mathrm{~cm}^{-1}$ red shift of the linearly bound CO stretching band, which now lies at $2032 \mathrm{~cm}^{-1}$. A shift is also observed for the PtC stretching mode, which is found at $484 \mathrm{~cm}^{-1}$.

Table 1 reports the mean CO stretching frequency and the corresponding average bond length obtained for the three considered coverages in both vacuum and water. At low coverage, the discussed red shift going from vacuum to liquid environment is associated with the elongation of the $\mathrm{C}-\mathrm{O}$ bond length. By increasing the coverage in the vacuum, the stretching band moves to higher frequencies, i.e., in the opposite direction, whereas in water, at the relatively high coverage of 0.50 , no significant frequency shift is observed with respect to the dry conditions.

In view of the previous structural analysis, we deduce that the presence of water interacting with the metal at $\Theta=0.25$ causes the red shift, which is then not due to a direct interaction between solvent and the CO adsorbates, e.g., through the formation of weak hydrogen bonds. ${ }^{14}$

In order to verify whether stable hydrogen bonds between water and $\mathrm{CO}$ are present, as previously suggested, ${ }^{11,14}$ we inspect the $\mathrm{O}-\mathrm{H}$ pair distribution function, $g_{\mathrm{OH}}(r)$, for the $\mathrm{O}$ atoms belonging to the $\mathrm{CO}$ adsorbates, and compared it to the same distribution computed for the $\mathrm{O}_{\text {wat }}$. The obtained $g_{\mathrm{OH}}(r)$ curves are reported in Fig. 7. It turns out that the closest $\mathrm{H}$ atoms to the $\mathrm{O}_{\mathrm{CO}}$ are at $2.8 \AA$, too far to form a hydrogen bond. Moreover, the feature at 2.8 
$\AA$ is mainly attributed to the water molecules of the bilayer. Indeed, it flattens significantly in the case of higher coverage, where the bi-layer has substantially vanished. These results seem to rule out a role of direct hydrogen bonding to $\mathrm{CO}$ to explain the modification of the IR spectra. Nevertheless, the presence of the correct dielectric environment above the adsorbates and the bi-layer is essential to reproduce the observed IR intensity enhancement (see Tab. 1).

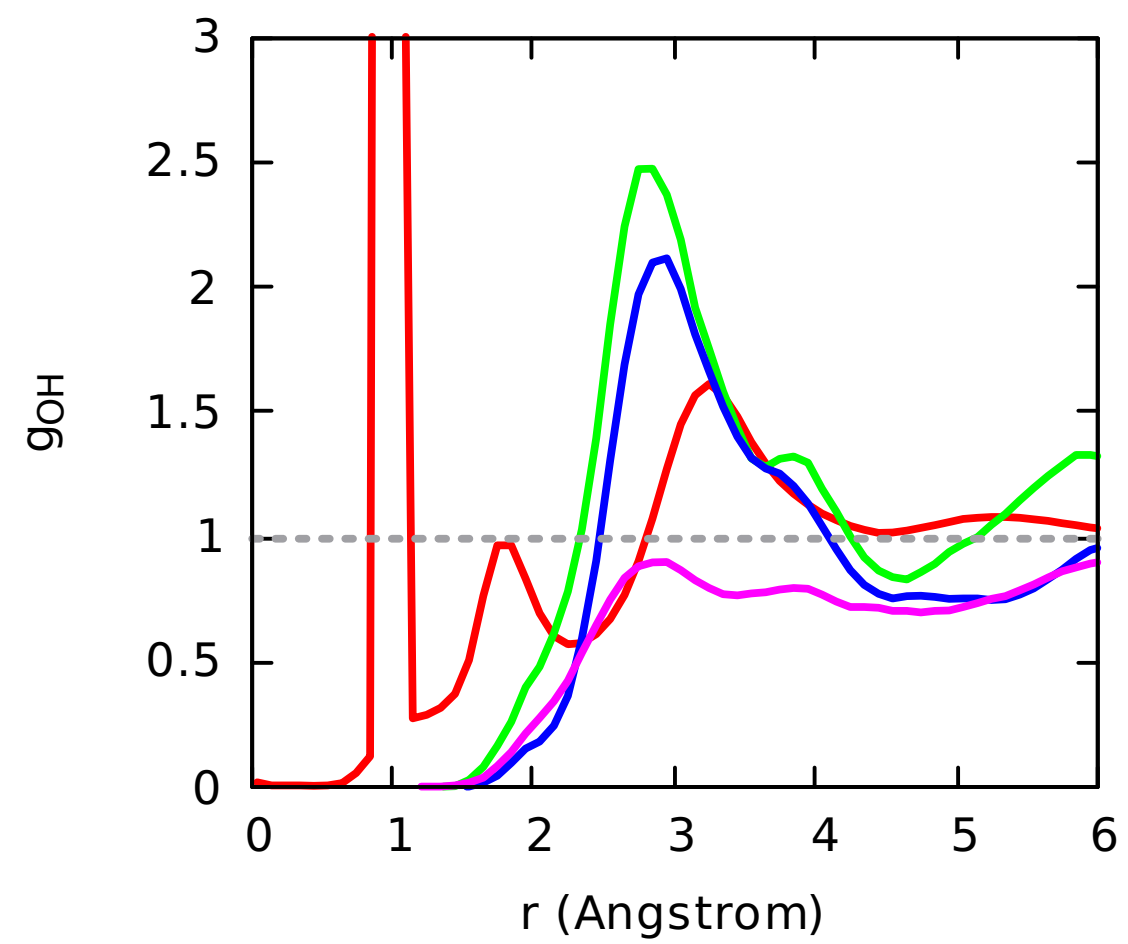

Figure 7: $\mathrm{O}-\mathrm{H}$ pair distribution function restricted to the water oxygen (red) or to the CO oxygen at coverage $\Theta=0.11$ (green), 0.25 (blue) and 0.50 (purple).

Our findings suggest that the red shift is an indirect effect of the chemisorption of water molecules of the first layer. In the vacuum, the $\mathrm{CO}$ is more strongly bound to the metal and the $\mathrm{Pt}-\mathrm{C}$ bond is more rigid, which also prevents the diffusion of bridged geometries. Increasing the coverage, by $\mathrm{CO}$ or $\mathrm{H}_{2} \mathrm{O}$ co-adsorbates, the strength of the adsorption is reduced, i.e., the $\mathrm{Pt}-\mathrm{C}$ bond is looser. This allows $\mathrm{CO}$ hopping events and determines the softer CO stretching mode. The given interpretation is also confirmed by the inspection of the 
Table 1: Vibrational frequencies and average bond length of linearly bound $\mathrm{CO}$ for the three considered coverages, in vacuum and in liquid environment. Frequencies and intensities obtained form CO coverage of 0.11 with different thickness of water layers. The spectra are provided in the supplementary information

\begin{tabular}{|c|c|c|c|c|c|}
\hline \multirow{2}{*}{ Coverage } & $v_{C O}$ & $\left(\mathrm{~cm}^{-1}\right)$ & \multicolumn{2}{|r|}{$d_{C O}$} & $(\AA)$ \\
\hline & vacuum & water & \multicolumn{2}{|c|}{ vacuum } & water \\
\hline 0.11 & 2048 & 2010 & \multicolumn{2}{|c|}{1.144} & 1.151 \\
\hline 0.25 & 2052 & 2032 & \multicolumn{2}{|c|}{1.144} & 1.150 \\
\hline \multirow[t]{2}{*}{0.50} & 2062 & 2061 & \multicolumn{2}{|c|}{1.145} & 1.148 \\
\hline & vacuum & 1 layer & 2 layers & 3 layers & water \\
\hline \multirow{2}{*}{ frequency $\left(\mathrm{cm}^{-1}\right)$} & 2048 & 2019 & 2008 & 2008 & 2010 \\
\hline & 0.29 & 0.28 & 0.30 & 0.40 & 0.67 \\
\hline
\end{tabular}

charge redistribution at the interface from both static calculations and molecular dynamics. For a detailed inspection of the electronic rearrangements associated with adsorption and co-adsorption, we have considered a simplified model. In particular, the structure of one CO adsorbate has been optimized, either alone, or in the presence of one and two co-adsorbed $\mathrm{H}_{2} \mathrm{O}$ molecules. For the $\mathrm{H}_{2} \mathrm{O}$ molecules, positions similar to those observed along the MD sampling have been taken as an initial guess. For each optimized structure, the vibrational analysis is performed within the harmonic approximation. The same trend in the frequency shift, as discussed above, is confirmed by the simplified model. The CO stretching frequency in vacuum turns out to be $2059 \mathrm{~cm}^{-1}$. By adding one co-adsorbed water molecule a red shift of $24 \mathrm{~cm}^{-1}$ is measured, while the second water molecule determines a red shift of 54 $\mathrm{cm}^{-1}$. The associated $\mathrm{C}-\mathrm{O}$ bond elongation is also in agreement with the MD results. The analysis of the electronic density rearrangement around the $\mathrm{CO}$ adsorbate shows that the presence of the water molecules induces a back transfer of electrons from the metal to the $\mathrm{O}_{\mathrm{CO}}$ (the charge density difference is reported in the Supporting Information), while there is no direct $\mathrm{CO} / \mathrm{H}_{2} \mathrm{O}$ interaction. The electron transfer from the Pt d-band to the anti-bonding $\mathrm{CO}$ molecular orbital is more pronounced because of the presence of the co-adsorbed water molecules, as also demonstrated by the increased population of the $2 \pi$ anti-bonding (see Fig.S9 in Supporting Information). This electron-redistribution weakens the C-O bond. 
The picture emerging from the static simplified model is consistent with the results extracted from the MD trajectory, by progressively increasing the number of water layers over the $\mathrm{CO} / \mathrm{Pt}$ sample, at constant coverage $\Theta=0.11$. The bottom part of Table 1 reports the mean $\mathrm{CO}$ stretching frequency and the corresponding IR intensity obtained including only one chemisorbed water layer, the full bi-layer, or also the third layer. It turns out, that the full bi-layer is needed to recover the correct red shift. However, the additional wetting layer is required to reproduce the IR intensity enhancement. This can be understood considering that the O-ends of the adsorbates are located within the depletion region of the water film, i.e., a partially positively charged region, as shown in Fig. 8. The resulting modulation in the electrostatic potential across the film enhances the transition dipole and explains the intensity increase.

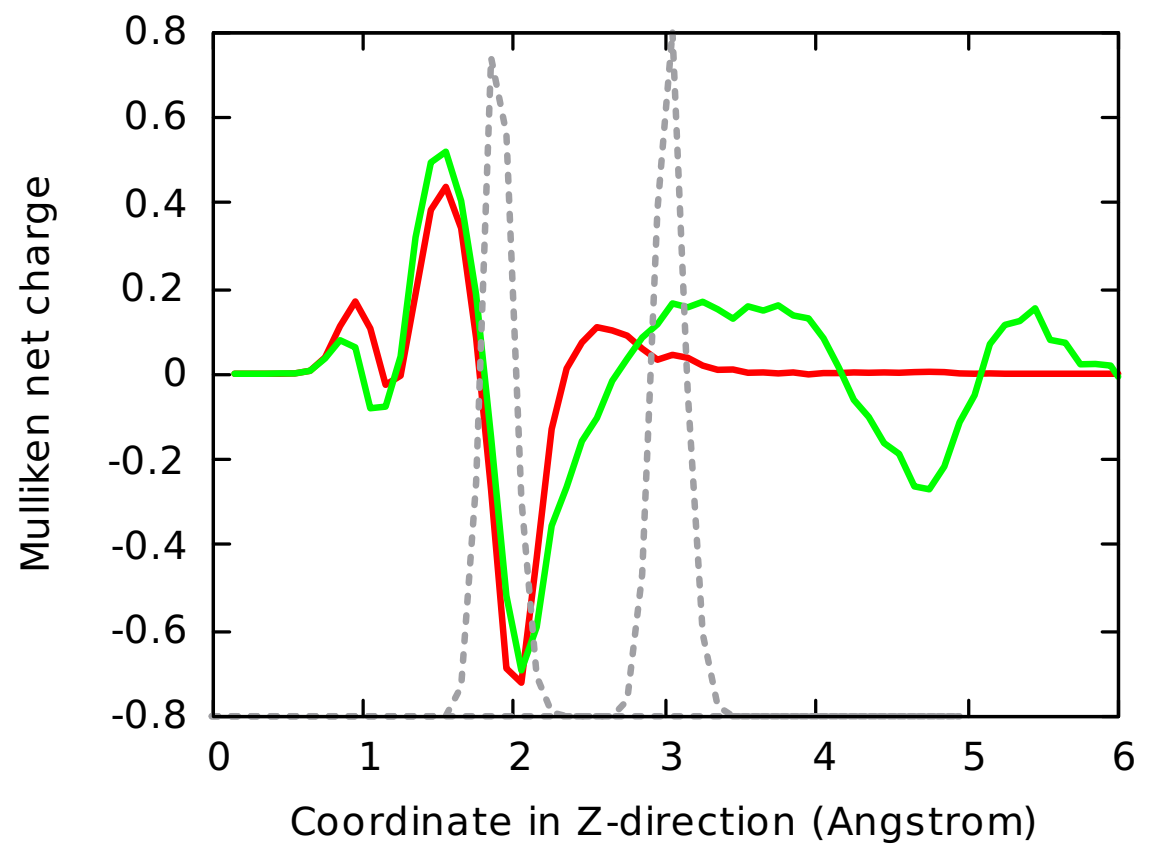

Figure 8: Mulliken net charge density as a function Z-coordinate of the bi-layer water (red line) and water film (green line), the dashedlines are the position of $\mathrm{CO}$ molecule.

The electronic charge redistribution along the MD trajectory can be efficiently monitored by means of a population analysis, e.g., by evaluating at each time step the Mulliken charges. The charge density computed from the Mulliken charges is then integrated within the planes 
parallel to the surface and averaged over the sampled configurations. The resulting profile along the surface normal can reveal charge transfers through the interface structure. Fig. S7 in Supporting Information displays such a profile computed for the solvated CO/Pt interface at the coverage of 0.25 . We observe the formation of the surface dipole with positive charge accumulation at the height of the $\mathrm{C}$ atoms and negative charge around the $\mathrm{O}$ atoms of the adsorbates.

By restricting the Mulliken density analysis to the water subsystem (trajectory without the presence of $\mathrm{CO} / \mathrm{Pt}$ ), as shown by the green line in Fig. S7 in Supporting Information, it appears that large part of the electronic rearrangement occurs within the water bi-layer, while the bulky part of the film is not significantly involved. Since the bi-layer water molecules transfer a certain amount of charge to the surface, electrons are back-donated to the nearby $\mathrm{CO}$ anti-bonding states, thus weakening the $\mathrm{CO}$ bond.

\section{Conclusions}

In this work, we used second-generation Car-Parrinello molecular dynamics to study the $\mathrm{Pt} / \mathrm{CO} /$ water interface at room temperature at different $\mathrm{CO}$ coverages. From the extensive sampling of the molecular configurations, relevant vibrational properties of the adsorbate have been investigated. We address the structural details of the solvent at the interface and correlate them to the adsorption geometry and to the dynamics of the $\mathrm{CO}$ molecules. In particular, we observe that the structured water bi-layer that is formed at the Pt-surface is progressively modified by increasing the $\mathrm{CO}$ coverage. In agreement with infra-red spectroscopy measurements in the aqueous phase, we could reproduce the frequency shift and the IR intensity enhancement for the samples at lower coverage, up to $\Theta=0.25$. The detailed analysis of the structure and of the electronic rearrangement at the interface reveals that the co-adsorption of water molecules and the consequent redistribution of the electrons among the $\mathrm{Pt} \mathrm{d}$-band and the $\mathrm{CO}$ antibonding states is at the origin of the CO-stretching 
frequency red shift. On the other hand, the water molecules over the $\mathrm{CO}$ adsorbate present a more liquid-like behaviour and do not directly interact with either the metal or the CO adsorbate. Nevertheless, the presence of the bulk liquid environment determines the characteristic electrostatic conditions that are at the origin of the transition dipole enhancement and IR intensity increase.

We have demonstrated that the proper modelling of such a complex interface by advanced AIMD techniques such as the SGCP approach is now feasible. The consideration of the electronic structure and the explicit solvent, together with the extended dynamical sampling under specific thermodynamic conditions, provide the required improved insight into the physics of the system at a molecular level. This approach is then suitable to address even more complex materials, helping to achieve a better understanding and control of their properties.

\section{Supporting Information Available}

The additional configurations, computational details, and charge analysis are given in Supporting Information.

This material is available free of charge via the Internet at http://pubs.acs.org/.

\section{Acknowledgement}

Discussions and suggestions from and David Paleček, Gökçen Tek and Gabriele Tocci are acknowledged. This work is funded by the SNF Sinergia grant and the University Research Priority Program (URPP) for solar light to chemical energy conversion (LightChEC) of the University of Zurich. The authors thank the generous allocation of computing resources from 
the Swiss National Supercomputing Center (CSCS) under project ID s657 and uzh1.

\section{References}

(1) Fujishima, A.; Honda, K. Electrochemical photolysis of water at a semiconductor electrode. nature 1972, 238, 37.

(2) Chen, X.; Shen, S.; Guo, L.; Mao, S. S. Semiconductor-based photocatalytic hydrogen generation. Chemical reviews 2010, 110, 6503-6570.

(3) Björneholm, O.; Hansen, M. H.; Hodgson, A.; Liu, L.-M.; Limmer, D. T.; Michaelides, A.; Pedevilla, P.; Rossmeisl, J.; Shen, H.; Tocci, G. Water at interfaces. Chemical reviews 2016, 116, 7698-7726.

(4) Pham, T. A.; Ping, Y.; Galli, G. Modelling heterogeneous interfaces for solar water splitting. Nature materials 2017, 16, 401.

(5) Yoon, Y.; Rousseau, R.; Weber, R. S.; Mei, D.; Lercher, J. A. First-principles study of phenol hydrogenation on $\mathrm{Pt}$ and Ni catalysts in aqueous phase. Journal of the American Chemical Society 2014, 136, 10287-10298.

(6) Rossmeisl, J.; Nørskov, J. K.; Taylor, C. D.; Janik, M. J.; Neurock, M. Calculated phase diagrams for the electrochemical oxidation and reduction of water over Pt (111). The Journal of Physical Chemistry B 2006, 110, 21833-21839.

(7) Yeh, K.-Y.; Wasileski, S. A.; Janik, M. J. Electronic structure models of oxygen adsorption at the solvated, electrified Pt (111) interface. Physical Chemistry Chemical Physics 2009, 11, 10108-10117.

(8) Herron, J. A.; Morikawa, Y.; Mavrikakis, M. Ab initio molecular dynamics of solvation effects on reactivity at electrified interfaces. Proceedings of the National Academy of Sciences 2016, 113, E4937-E4945. 
(9) Miki, A.; Ye, S.; Osawa, M. Surface-enhanced IR absorption on platinum nanoparticles: an application to real-time monitoring of electrocatalytic reactions. Chemical Communications 2002, 1500-1501.

(10) Lu, G.; Lagutchev, A.; Dlott, D. D.; Wieckowski, A. Quantitative vibrational sumfrequency generation spectroscopy of thin layer electrochemistry: CO on a Pt electrode. Surface Science 2005, 585, 3-16.

(11) Xiong, W.; Laaser, J. E.; Mehlenbacher, R. D.; Zanni, M. T. Adding a dimension to the infrared spectra of interfaces using heterodyne detected 2D sum-frequency generation (HD 2D SFG) spectroscopy. Proceedings of the National Academy of Sciences 2011, 108, 20902-20907.

(12) Kraack, J. P.; Lotti, D.; Hamm, P. Ultrafast, multidimensional attenuated total reflectance spectroscopy of adsorbates at metal surfaces. The journal of physical chemistry letters 2014, 5, 2325-2329.

(13) Ebbesen, S. D.; Mojet, B. L.; Lefferts, L. CO Adsorption and Oxidation at the Catalyst- Water Interface: An Investigation by Attenuated Total Reflection Infrared Spectroscopy. Langmuir 2006, 22, 1079-1085.

(14) Ebbesen, S. D.; Mojet, B. L.; Lefferts, L. In situ ATR-IR study of CO adsorption and oxidation over $\mathrm{Pt} / \mathrm{Al} 2 \mathrm{O} 3$ in gas and aqueous phase: promotion effects by water and pH. Journal of Catalysis 2007, 246, 66-73.

(15) Mojet, B. L.; Ebbesen, S. D.; Lefferts, L. Light at the interface: the potential of attenuated total reflection infrared spectroscopy for understanding heterogeneous catalysis in water. Chemical Society Reviews 2010, 39, 4643-4655.

(16) Feibelman, P. J.; Hammer, B.; Nørskov, J. K.; Wagner, F.; Scheffler, M.; Stumpf, R.; Watwe, R.; Dumesic, J. The CO/Pt (111) puzzle. The Journal of Physical Chemistry B 2001, 105, 4018-4025. 
(17) Wang, Y.; de Gironcoli, S.; Hush, N. S.; Reimers, J. R. Successful a priori modeling of $\mathrm{CO}$ adsorption on pt (111) using periodic hybrid density functional theory. Journal of the American Chemical Society 2007, 129, 10402-10407.

(18) Koper, M. T.; van Santen, R. A.; Wasileski, S. A.; Weaver, M. J. Field-dependent chemisorption of carbon monoxide and nitric oxide on platinum-group (111) surfaces: Quantum chemical calculations compared with infrared spectroscopy at electrochemical and vacuum-based interfaces. The Journal of Chemical Physics 2000, 113, 4392-4407.

(19) Villegas, I.; Weaver, M. J. Carbon monoxide adlayer structures on platinum (111) electrodes: A synergy between in-situ scanning tunneling microscopy and infrared spectroscopy. The Journal of chemical physics 1994, 101, 1648-1660.

(20) Kizhakevariam, N.; Jiang, X.; Weaver, M. J. Infrared spectroscopy of model electrochemical interfaces in ultrahigh vacuum: The archetypical case of carbon monoxide/water coadsorption on Pt (111). The Journal of chemical physics 1994, 100, 67506764 .

(21) Steininger, H.; Lehwald, S.; Ibach, H. On the adsorption of CO on Pt (111). Surface Science 1982, 123, 264-282.

(22) Hamm, P.; Zanni, M. Concepts and methods of 2D infrared spectroscopy; Cambridge University Press, 2011.

(23) Severson, M. W.; Stuhlmann, C.; Villegas, I.; Weaver, M. J. Dipole-dipole coupling effects upon infrared spectroscopy of compressed electrochemical adlayers: Application to the Pt (111)/CO system. The Journal of chemical physics 1995, 103, 9832-9843.

(24) Mamatkulov, M.; Filhol, J.-S. An ab initio study of electrochemical vs. electromechanical properties: the case of $\mathrm{CO}$ adsorbed on a Pt (111) surface. Physical Chemistry Chemical Physics 2011, 13, 7675-7684. 
(25) Dabo, I.; Cancès, E.; Li, Y.; Marzari, N. Towards first-principles electrochemistry. arXiv preprint arXiv:0901.0096 2008,

(26) Janik, M. J.; Neurock, M. A first principles analysis of the electro-oxidation of CO over Pt (1 1 1). Electrochimica acta 2007, 52, 5517-5528.

(27) Iyemperumal, S. K.; Deskins, N. A. Evaluating Solvent Effects at the Aqueous/Pt (111) Interface. ChemPhysChem 2017, 18, 2171-2190.

(28) Duan, S.; Xu, X.; Luo, Y.; Hermansson, K.; Tian, Z.-Q. Thermal effects on electronic properties of $\mathrm{CO} / \mathrm{Pt}$ (111) in water. Physical Chemistry Chemical Physics 2013, 15, $13619-13627$.

(29) Schnur, S.; Groß, A. Properties of metal-water interfaces studied from first principles. New Journal of Physics 2009, 11, 125003.

(30) Le, J.; Iannuzzi, M.; Cuesta, A.; Cheng, J. Determining Potentials of Zero Charge of Metal Electrodes versus the Standard Hydrogen Electrode from Density-FunctionalTheory-Based Molecular Dynamics. Physical Review Letters 2017, 119, 016801.

(31) Bouzid, A.; Pasquarello, A. Atomic-Scale Simulation of Electrochemical Processes at Electrode/Water Interfaces under Referenced Bias Potential. The journal of physical chemistry letters 2018, 9, 1880-1884.

(32) Groß, A. Ab initio molecular dynamics simulations of the $\mathrm{O} 2 / \mathrm{Pt}$ (111) interaction. Catalysis Today 2016, 260, 60-65.

(33) Kühne, T. D.; Krack, M.; Mohamed, F. R.; Parrinello, M. Efficient and accurate CarParrinello-like approach to Born-Oppenheimer molecular dynamics. Physical review letters 2007, 98, 066401.

(34) Kühne, T. D. Second generation Car-Parrinello molecular dynamics. Wiley Interdisciplinary Reviews: Computational Molecular Science 2014, 4, 391-406. 
(35) Hutter, J. Car-Parrinello molecular dynamics. Wiley Interdisciplinary Reviews: Computational Molecular Science 2012, 2, 604-612.

(36) Hutter, J.; Iannuzzi, M.; Schiffmann, F.; VandeVondele, J. CP2K: atomistic simulations of condensed matter systems. Wiley Interdisciplinary Reviews: Computational Molecular Science 2014, 4, 15-25.

(37) VandeVondele, J.; Krack, M.; Mohamed, F.; Parrinello, M.; Chassaing, T.; Hutter, J. Quickstep: Fast and accurate density functional calculations using a mixed Gaussian and plane waves approach. Computer Physics Communications 2005, 167, 103-128.

(38) VandeVondele, J.; Hutter, J. Gaussian basis sets for accurate calculations on molecular systems in gas and condensed phases. The Journal of chemical physics 2007, 127, 114105.

(39) Goedecker, S.; Teter, M.; Hutter, J. Separable dual-space Gaussian pseudopotentials. Physical Review B 1996, 54, 1703.

(40) Hartwigsen, C.; Gœdecker, S.; Hutter, J. Relativistic separable dual-space Gaussian pseudopotentials from H to Rn. Physical Review B 1998, 58, 3641.

(41) Alaei, M.; Akbarzadeh, H.; Gholizadeh, H.; de Gironcoli, S. C O/ Pt (111): GGA density functional study of site preference for adsorption. Physical Review B 2008, 77, 085414.

(42) Kresse, G.; Gil, A.; Sautet, P. Significance of single-electron energies for the description of CO on Pt (111). Physical Review B 2003, 68, 073401.

(43) Stroppa, A.; Termentzidis, K.; Paier, J.; Kresse, G.; Hafner, J. CO adsorption on metal surfaces: A hybrid functional study with plane-wave basis set. Physical Review B 2007, 76,195440 . 
(44) Wang, Y.; de Gironcoli, S.; Hush, N. S.; Reimers, J. R. Successful a priori modeling of CO adsorption on pt (111) using periodic hybrid density functional theory. Journal of the American Chemical Society 2007, 129, 10402-10407.

(45) Orita, H.; Itoh, N.; Inada, Y. All electron scalar relativistic calculations on adsorption of $\mathrm{CO}$ on $\mathrm{Pt}$ (111) with full-geometry optimization: a correct estimation for CO sitepreference. Chemical physics letters 2004, 384, 271-276.

(46) Schimka, L.; Harl, J.; Stroppa, A.; Grüneis, A.; Marsman, M.; Mittendorfer, F.; Kresse, G. Accurate surface and adsorption energies from many-body perturbation theory. Nature Materials 2010, 9, 741-744.

(47) Sun, J.; Marsman, M.; Ruzsinszky, A.; Kresse, G.; Perdew, J. P. Improved lattice constants, surface energies, and CO desorption energies from a semilocal density functional. Physical Review B 2011, 83, 121410.

(48) Becke, A. D. Density-functional thermochemistry. I. The effect of the exchange-only gradient correction. The Journal of chemical physics 1992, 96, 2155-2160.

(49) Lee, C.; Yang, W.; Parr, R. G. Development of the Colle-Salvetti correlation-energy formula into a functional of the electron density. Physical review B 1988, 37, 785.

(50) Goerigk, L.; Kruse, H.; Grimme, S. Benchmarking Density Functional Methods against the S66 and S66x8 Datasets for Non-Covalent Interactions. ChemPhysChem 2011, 12, $3421-3433$.

(51) Del Ben, M.; SchÃúnherr, M.; Hutter, J.; VandeVondele, J. Bulk liquid water at ambient temperature and pressure from MP2 theory. The journal of physical chemistry letters 2013, 4, 3753-3759.

(52) Del Ben, M.; Hutter, J.; VandeVondele, J. Probing the structural and dynamical prop- 
erties of liquid water with models including non-local electron correlation. The Journal of chemical physics 2015, 143, 054506.

(53) Kolafa, J. Time-reversible always stable predictor-corrector method for molecular dynamics of polarizable molecules. Journal of computational chemistry 2004, 25, 335-342.

(54) VandeVondele, J.; Hutter, J. An efficient orbital transformation method for electronic structure calculations. The Journal of chemical physics 2003, 118, 4365-4369.

(55) Thomas, M.; Brehm, M.; Fligg, R.; Vöhringer, P.; Kirchner, B. Computing vibrational spectra from ab initio molecular dynamics. Physical Chemistry Chemical Physics 2013, 15, 6608-6622.

(56) Brehm, M.; Kirchner, B. TRAVIS-a free analyzer and visualizer for Monte Carlo and molecular dynamics trajectories. 2011.

(57) King-Smith, R.; Vanderbilt, D. Theory of polarization of crystalline solids. Physical Review $B$ 1993, 47, 1651.

(58) Resta, R. Macroscopic polarization in crystalline dielectrics: the geometric phase approach. Reviews of modern physics 1994, 66, 899.

(59) Marx, D.; Hutter, J. Ab initio molecular dynamics. Parallel computing 2009, 309, 327.

(60) Wang, J. H. Self-diffusion coefficients of water. The Journal of Physical Chemistry 1965, 69, 4412-4412.

(61) Meng, S.; Wang, E.; Gao, S. Water adsorption on metal surfaces: A general picture from density functional theory studies. Physical Review B 2004, 69, 195404.

(62) Kühne, T. D.; Pascal, T. A.; Kaxiras, E.; Jung, Y. New insights into the structure of the vapor/water interface from large-scale first-principles simulations. The journal of physical chemistry letters 2010, 2, 105-113. 
Graphical TOC Entry

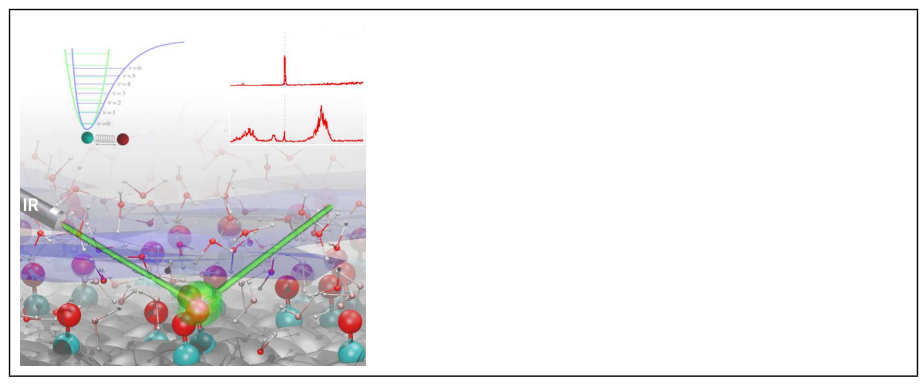

